\title{
IMPROVEMENT OF THE REALIGNMENT PERFORMANCE FOR SHORT WAVELENGTH TRACK IRREGULARITY ON A TAMPING MACHINE
}

\author{
TARO YADA, YOSHINOBU SODA \& YASUKUNI NAGANUMA \\ Technology Research and Development Department, Central Japan Railway Company, Japan
}

\begin{abstract}
Track maintenance work is essential to maintaining the safe and stable operation of a railway system. In recent years, the realignment of track irregularity with wavelengths of approximately $5 \mathrm{~m}$ is required in order to reduce the Q-force and the track irregularity growth on the Tokaido Shinkansen. Tamping machines are generally used to realign track irregularities. But, the newest Type- 09 tamping machine has a lower realignment performance for track irregularities of around $5 \mathrm{~m}$ in wavelength due to its capabilities when it comes to measuring chord length. Therefore, a new measuring system has been attached in the tamping machine in order to measure $3.6 \mathrm{~m}$ versine at the longitudinal level. Since a high-precision gyroscope is used, the measuring system is not influenced by the vibration of the tamping machine when in operation. Through repeatability testing it was confirmed that short wavelength track irregularities could be measured with high accuracy. The data measured by Dr Yellow (a track inspection car) was combined with the data taken from the gyroscope to propose a new algorithm for correcting $5 \mathrm{~m}$ chord versine track irregularities with better accuracy. Maintenance tests will be conducted to verify the results.
\end{abstract}

Keywords: tamping machine, gyroscope, short wavelength, track irregularity.

\section{INTRODUCTION}

The Tokaido Shinkansen is an important transportation route in Japan between Tokyo and Shin-Osaka that has had no accidents resulting in fatalities or injuries since the commencement of operation in 1964 thus making it the most unique rapid transit railway in the world.

In March 2015, the maximum speed of the Tokaido Shinkansen was increased to $285 \mathrm{kpm}$ from the previous maximum speed of $270 \mathrm{kpm}$. In the future, we will continue to improve the transport service of the Tokaido Shinkansen.

Track maintenance is essential to maintain the safe and stable operation of a railway. Track maintenance using a tamping machine is widely performed in various countries as an effective track maintenance method.

In order to maintain Tokaido Shinkansen tracks with high accuracy a reverse filter is used to calculate track irregularities. Precise adjustment of position is essential for maximum utilization. However, the Type-09 tamping machine has lower realignment performance for track irregularities around wavelengths of $5 \mathrm{~m}$ due to its capability to measure chord lengths. This research introduces a new algorithm that can realign track irregularities to a wavelength of about $5 \mathrm{~m}$ with high accuracy on tamping machines and the various tests that were conducted [1].

\section{THE CORRELATION BETWEEN WHEEL LOAD VARIATION AND TRACK IRREGULARITY}

Track maintenance techniques are important for restraining wheel load variation, preventing the degradation and destruction of track materials, and decreasing environmental load (noise and vibration) in order to maintain a high-quality service offered by high-speed railways. 
Fig. 1 shows wheel load fluctuations measured using the Tokaido Shinkansen prototype (hereinafter referred to as "X0 Trainset"). Fig. 2 and Fig. 3 show track irregularities for $5 \mathrm{~m}$ chord versine and $10 \mathrm{~m}$ chord versine detected by a track inspection car (Dr Yellow). The value of $5 \mathrm{~m}$ chord versine track irregularities is high for areas with relatively high wheel load showing stronger correlation compared with $10 \mathrm{~m}$ chord versine irregularities. So, in order to suppress wheel load, it is important to re-align track $5 \mathrm{~m}$ chord versine irregularities [1].

\section{TRACK MAINTENANCE BY USING TAMPING MACHINE ON TOKAIDO SHINKANSEN}

The majority of the track structures on Tokaido Shinkansen comprise ballast track, such as embankments and undercut structures. Therefore, track maintenance using a tamping machine has become mainstream. When using a tamping machine, the amount of track lift is calculated from the original waveform restored based on measurement data from Dr Yellow.

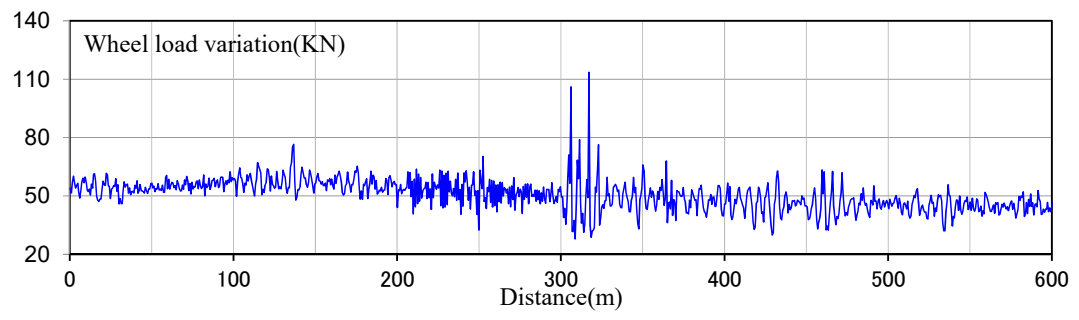

Figure 1: Wheel load obtained by the preceding experimental Tokaido-Shinkansen.

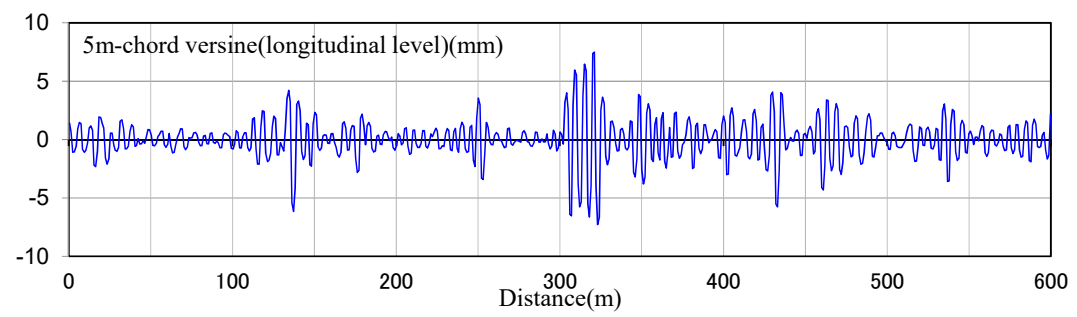

Figure 2: $5 \mathrm{~m}$ chord versine track irregularities detected by Dr Yellow.

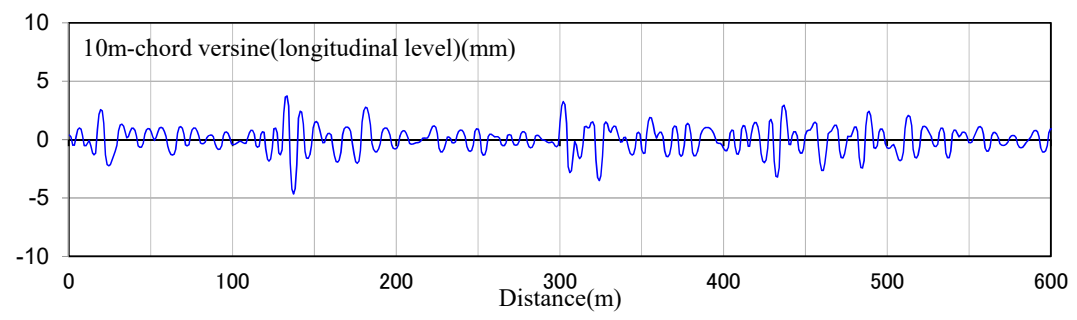

Figure 3: $10 \mathrm{~m}$ chord versine track irregularities detected by Dr Yellow. 
However, since Dr Yellow is only run once every 10 days, the progression of the detected irregularity between the time it is detected and the day of maintenance is not considered. Therefore, there is the possibility that the position measured by Dr Yellow and the position realigned by the tamping machine are not exact.

An automatic guide computer device called the 'Win-ALC' is installed in the Type-09 tamping machine (Fig. 4).

This device has function for inputting the amount of track lift calculated using measurement data from Dr Yellow in advance, and also ascertaining track irregularities in real time from the tamping machine's track measurement equipment. Utilizing the Win-ALC enables us to perform track maintenance with higher accuracy than before [1].

\section{TRACK IRREGULARITY MEASUREMENT ATTRIBUTES OF THE TYPE-09 AND TYPE-08 TAMPING MACHINES}

Fig. 5 shows the chord length measurement method of the Type-09 tamping machine. A wire is tied to three points; the front, middle and rear feelers. The Type-09 tamping machine can measure vertical irregularities for an asymmetrical chord offset ratio of 2:1. Fig. 6 shows the chord length measurement method of the Type- 08 tamping machine. The Type- 08 tamping machine can measure vertical irregularities for an asymmetrical chord offset ratio of $3: 1$ [1].

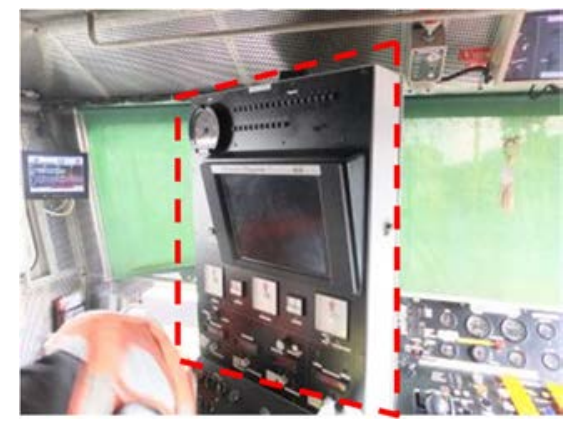

Figure 4: Automatic guide computer device called the 'Win-ALC'.

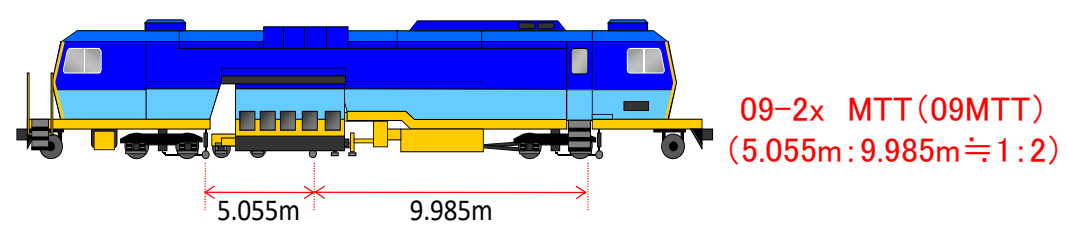

Figure 5: The chord length measurement method of the Type-09 tamping machine.

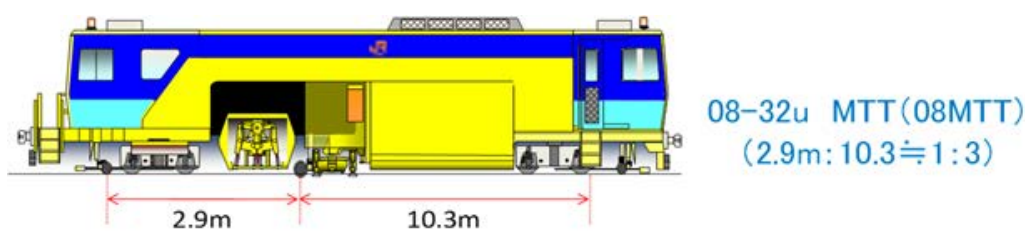

Figure 6: The chord length measurement method of the Type-08 tamping machine. 
In Fig. 7, the track shape on the ground is $x(\xi)$, and the wave form of the asymmetrical chord offset obtained through measurement is $y(\xi)$. The track inspection measurement by the tamping machine is represented by eqn (1).

$$
y(\xi)=x(\xi)-\{\beta x(\xi-a)+\alpha x(\xi+b)\} .
$$

At this point,

$$
\alpha=\frac{a}{a+b-c}, \beta=\frac{b-c}{a+b-c} .
$$

Here a Fourier conversion is applied to both sides of eqn (1),

$$
Y(\omega)=X(\omega)-\left\{\beta X(\omega) e^{-j \omega a}+\alpha X(\omega) e^{j \omega b}\right\}
$$

and, as a result, frequency attributes of the tamping machine are represented by eqn (3),

$$
H(\omega)=\frac{Y(\omega)}{X(\omega)}=1-\left\{\beta e^{-j \omega a}+\alpha e^{j \omega b}\right\} .
$$

Fig. 8 shows the inspection attributes of the Type- 09 and -08 tamping machines. The horizontal axis shows the space frequency of track irregularities, and the vertical axis shows amplitude gain. The characteristics of inspected track irregularities of the Type-09 tamping machine disappear around the wavelength of $5 \mathrm{~m}$.

So, the Type-09 tamping machine doesn't have the sufficient ability to realign track irregularities around the wavelength of $5 \mathrm{~m} \mathrm{[1],} \mathrm{[2].}$

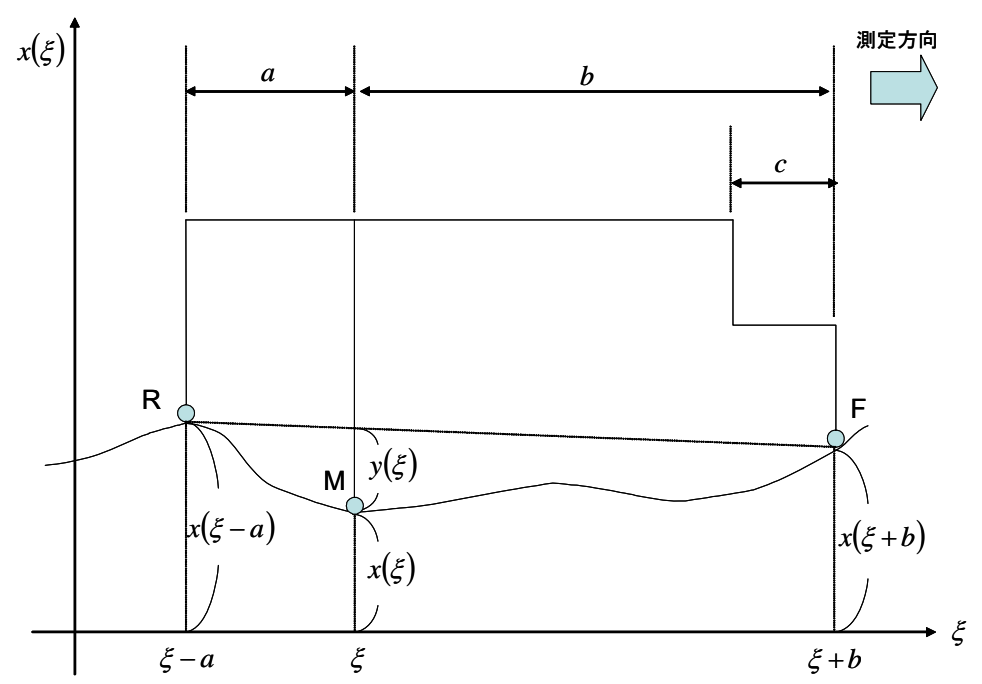

Figure 7: The measurement mechanism on the tamping machine. 


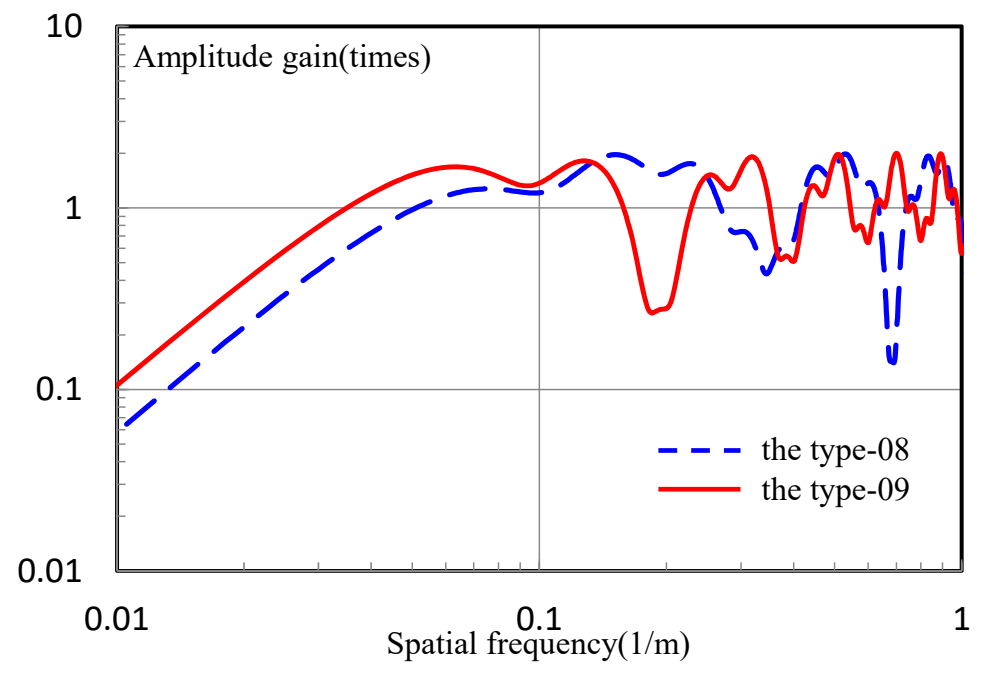

Figure 8: The characteristics of inspected track irregularity of the Type-09 and -08 tamping machines.

\section{INSTALLATION OF A GYROSCOPE IN THE TYPE-09 TAMPING MACHINE}

We have installed a gyroscope in the Type-09 tamping machine in order to measure track irregularities with a wavelength of around $5 \mathrm{~m}$. Fig. 9 shows the gyroscope installed in the Type-09 tamping machine. The gyroscope is installed between the axle boxes, which are $1.8 \mathrm{~m}$ apart, on the forward bogie of the tamping machine in order to measure track irregularity with a $1.8-\mathrm{m}$ chord length.

\section{TRACK IRREGULARITY INSPECTION ATTRIBUTES USING THE GYROSCOPE}

We chose the primary calculus of difference that could be measured from the two points measures using the gyroscope principle (Fig. 10).

The calculus of difference method is a way to measure the difference in elevation y $(\xi)$ between two points (L) at the actual shape of the track $\mathrm{x}(\xi)$ using the angle of inclination $\theta$. Since $\theta$ is minute, $\mathrm{I} \fallingdotseq \mathrm{L}$.

$$
y(\xi)=x(\xi)-x(\xi-l) \cong L \tan \theta
$$

Fourier conversion is shown in eqn (4). Eqn (5) shows the frequency attributes for the primary calculus of difference.

$$
H_{F D}(\omega)=Y(\omega) / X(\omega)=1-e^{-j \omega L} .
$$

Fig. 11 shows amplitude attributes when $\mathrm{L}=1.8 \mathrm{~m}$. The gyroscope superior capability for detecting $5 \mathrm{~m}$ chord versine track irregularities. 

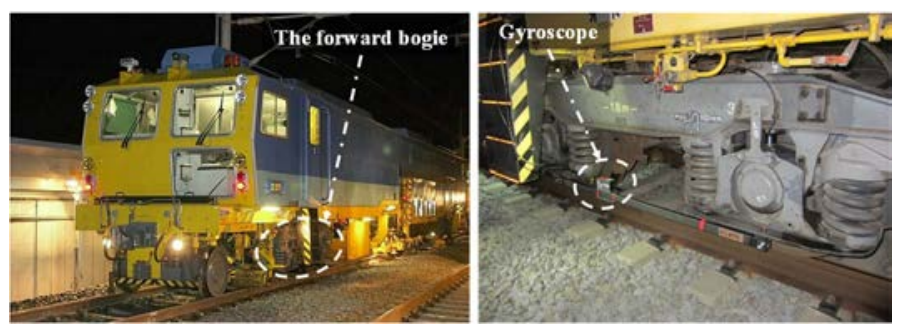

Figure 9: Gyroscope installation.

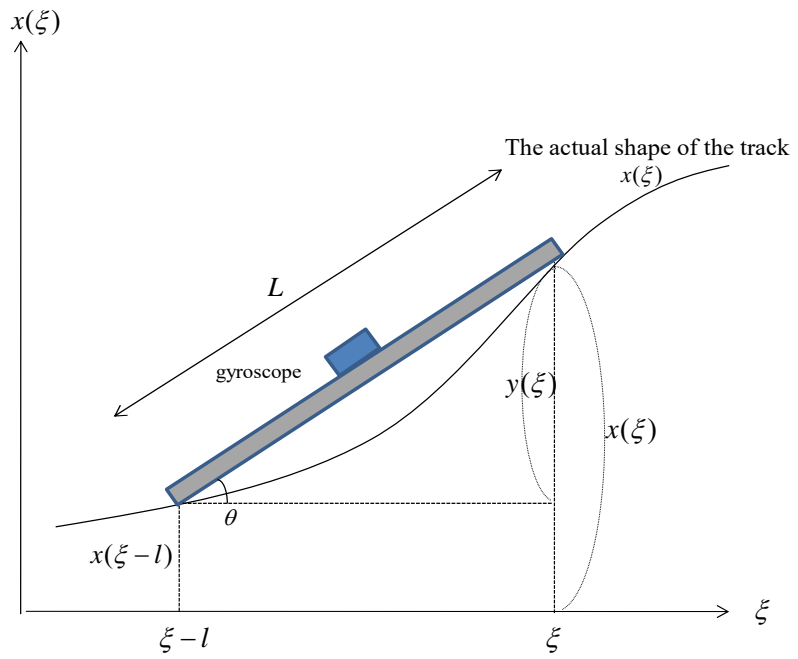

Figure 10: Primary calculus of difference for gyroscope measurement principle.

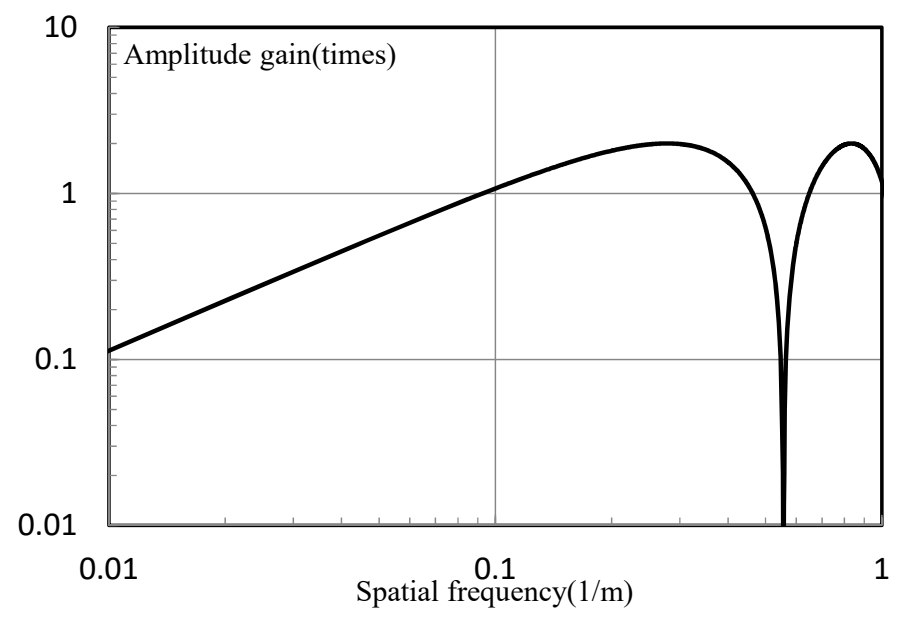

Figure 11: Gyroscope inspection attributes. 


\section{VERIFICATION TESTING FOR SHORT WAVELENGTH TRACK IRREGULARITIES ( AT THE MAINTENANCE DEPOT)}

We performed tests to verify whether or not short wavelength irregularities could be measured using the gyroscope installed in the tamping machine. The tests were performed in the Tokaido Shinkansen maintenance depot. Firstly, we measured the track irregularity using a portable track inspection trolley. This inspection trolley can measure short wavelength track irregularities down to $2.5 \mathrm{~m}$ chord versine accurately. We then compared the track irregularity measured with the portable track inspection trolley with the track irregularity measured by the gyroscope installed in the tamping machine, and confirmed the accuracy of measurement by the gyroscope.

In this instance, as shown in Fig. 12, one, three and then five track sleepers were removed from a section of track in the maintenance depot to reproduce predicted short wavelength track irregularities. For measurement runs were made, if you include the control run with no sleepers removed, with the tamping machine accelerating and decelerating.

\subsection{The results of the verification test (at the maintenance depot)}

$2.5 \mathrm{~m}$ chord versine irregularities measured both forward and backward were compared to verify reproducibility by the gyroscope. The comparison is shown in Fig. 13. The difference between forward and backward measurements is negligible thereby showing sufficient reproducibility. The measurements taken with the portable inspection trolley were also overlaid and were found to match the results from the gyroscope. Since the portable inspection trolley does not weigh as much it could not detect track irregularities at the location of the missing track sleepers, so the measurement taken with all sleepers intact was used for comparison. That comparison is shown in Fig. 14. Both waveforms matched and the margin of error was less than $2 \mathrm{~mm}$.

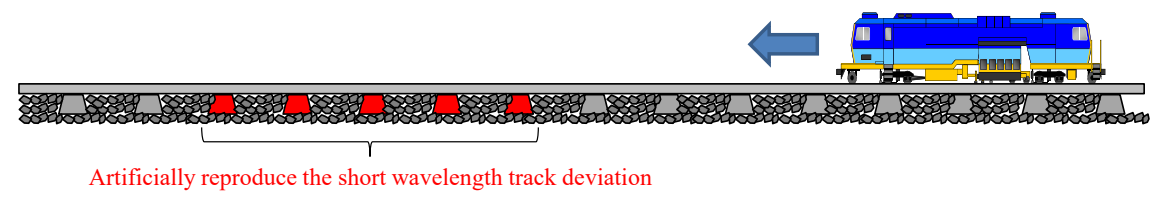

Figure 12: Overview of the verification test for the gyroscope.

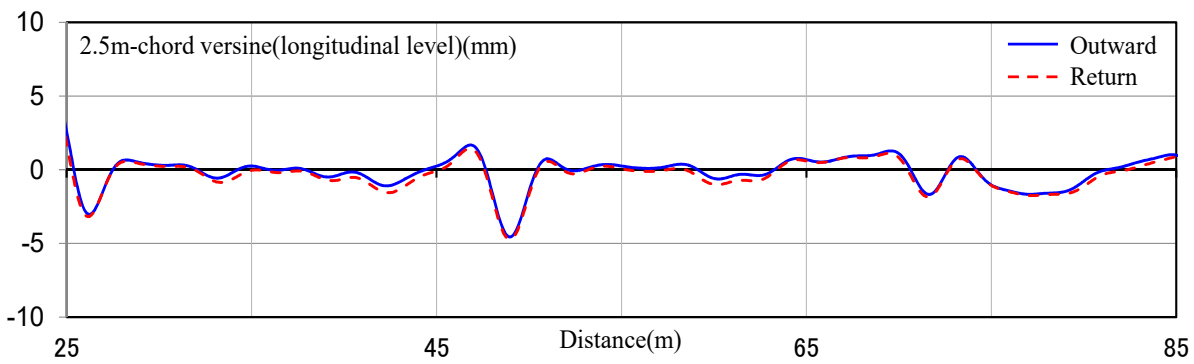

Figure 13: The reproducibility of gyroscope (at the maintenance depot). 


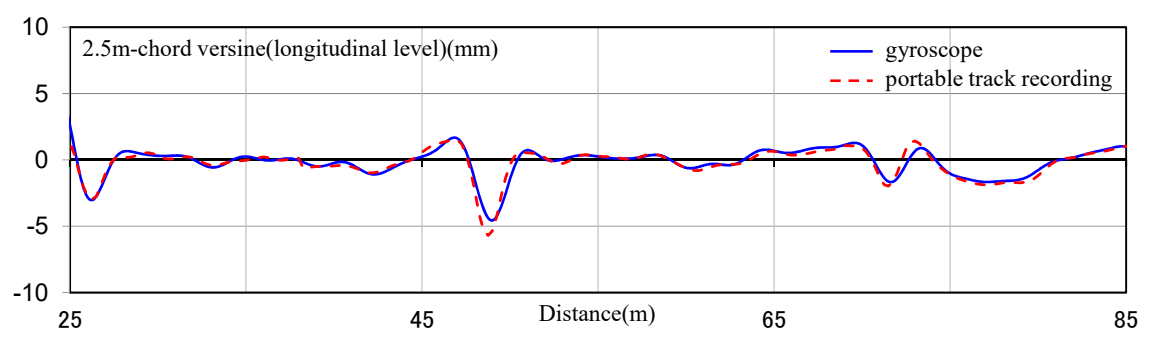

Figure 14: The consistency of the gyroscope (at the maintenance depot).

\section{VERIFICATION TESTING FOR SHORT WAVELENGTH TRACK IRREGULARITIES (ON THE MAIN LINE)}

Since verification tests at the maintenance depot showed that the gyroscope has sufficient consistency and reproducibility, tests on short wavelength irregularities were conducted on the main line. Measurement tests on the main line were performed during repeated accelerations and decelerations.

\subsection{The results of the verification tests (on the main line)}

The verification test results are shown in Fig. 15. A comparison of short-wavelength track irregularity data from going forward and backward verified that measurements on the main line have sufficient reproducibility. Further, the data difference between the two was $0.5 \mathrm{~mm}$ or less, even when the tamping machine was accelerating and decelerating, thereby verifying that short-wavelength track irregularities can be measured with high accuracy.

\section{PROPOSAL OF NEW TRACK MAINTENANCE TECHNIQUE USING A TAMPING MACHINE}

\subsection{Procedure of technique}

A method of track maintenance that uses a tamping machine and combines the data measured by Dr Yellow with data measured using a gyroscope was examined. When using only data measured by the gyroscope the accuracy of long wavelength track irregularities is poor which

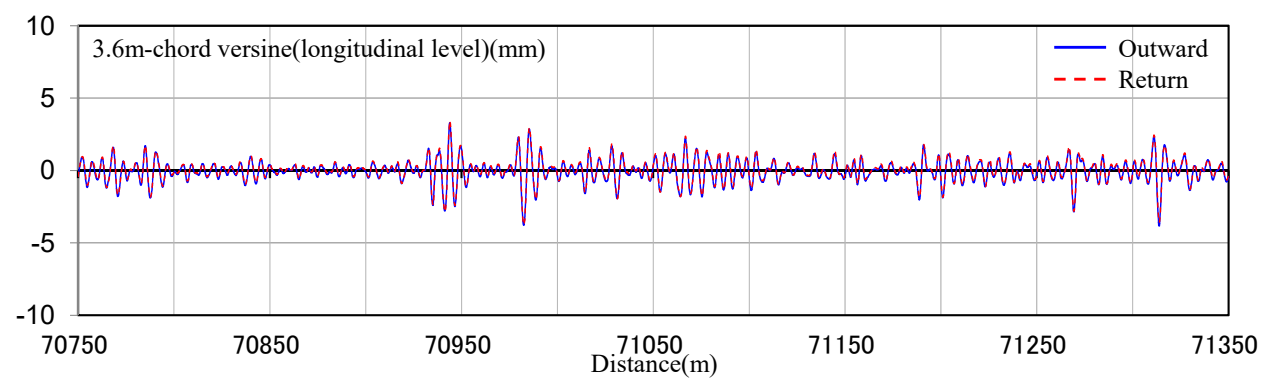

Figure 15: The reproducibility of gyroscope (on the main line). 
can lead to decreased ride comfort. Therefore, the long wavelength track irregularity data is taken from Dr yellow and short wavelength irregularity data is taken from the gyroscope. The procedure is as follows:

(1) Calculate a restored waveform treated with $2.5 \mathrm{~m}-3 \mathrm{~m}-100 \mathrm{~m}-150 \mathrm{~m}$ of BPF using the wavelength band from the data measured by Dr Yellow.

(2) Create repair plan line from (1).

(3) Calculate track irregularity from (1) by dividing the $3.6 \mathrm{~m}$ chord by 2.

(4) Subtract (1) from (3).

(5) On site, use the gyroscope to measure and calculate the track irregularity by dividing the $3.6 \mathrm{~m}$ chord by 2 .

(6) Use (5) for calibration, add (5) to (4) and perform maintenance.

9.2 Testing of new track maintenance technique using a tamping machine

Fig. 16 shows a comparison of before and after the test. You can see that both $5 \mathrm{~m}$ and $10 \mathrm{~m}$ chord track irregularities have been largely improved. Short wavelength irregularities that conventionally have not been able to be improved were improved using this method.

\section{CONCLUSIONS}

A) It was possible to measure short-wavelength track irregularities by using a gyroscope in a tamping machine.

B) Acceleration and deceleration of the tamping machine had no impact on measurements and short wavelength irregularities were able to be detected.

C) Combining the data measured with the gyroscope and that measure by Maya enabled short wavelength irregularities to be corrected during testing.

\section{FUTURE DEVELOPMENT}

A) Deliberate and create specifications for an Automatic MTT Realignment System.

B) Perform tests using the constructed Automatic MTT Realignment System and the selected gyroscope.

C) Perform an equal amount of long wavelength irregularity corrections as conventional methods in order to determine if the new method results in the same performance as the conventional method.

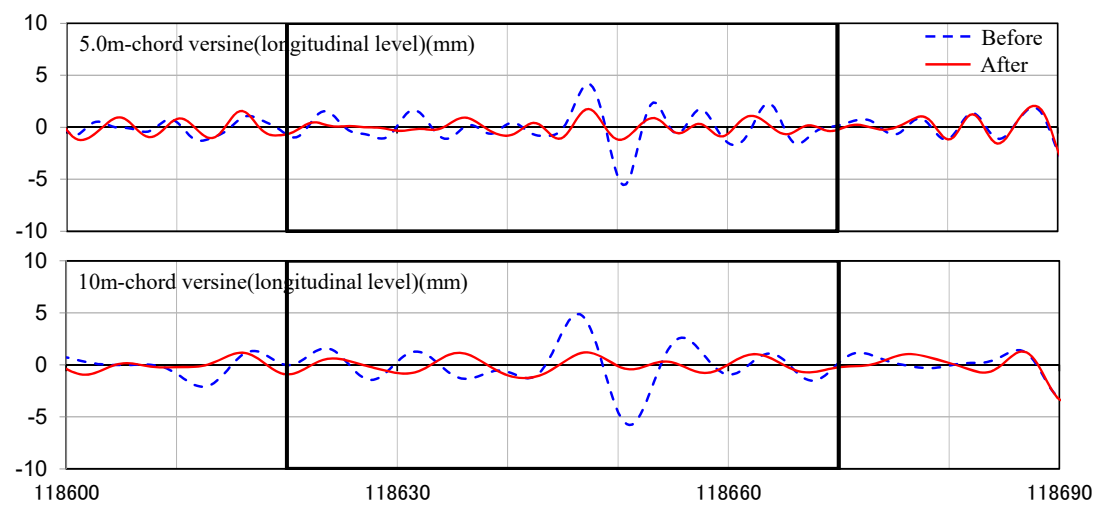

Figure 16: Test construction results. 
70 Computational Methods and Experimental Measurements XVIII

\section{REFERENCES}

[1] Aoki, T. \& Naganuma, Y., Improvement of the re-alignment performance for short wavelength track irregularity on tamping machine. Railway Engineering, 2015.

[2] Okumura, T. \& Naganuma, Y., Inspection accuracy of the tamping machine. 18th Japan Society of Civil Engineering, 2008. 